\title{
Automatic Malignant Thyroid Nodule Recognition in Ultrasound Images based on Deep Learning
}

\author{
Meng Zhou ${ }^{1}$, Rui Wang ${ }^{1, *}$, Peng Fu², Yang $\mathrm{Bai}^{3}$ and Ligang $\mathrm{Cui}^{2}$ \\ ${ }^{1}$ Key Laboratory of Precision Opto-mechatronics Technology Opto-eletronics Engineering, Beihang University Beijing, China \\ ${ }^{2}$ Department of Ultrasound, Peking University Third Hospital, Beijing, China. \\ ${ }^{3}$ Department of general surgery, Peking University Third Hospital.
}

\begin{abstract}
As the most common malignancy in the endocrine system, thyroid cancer is usually diagnosed by discriminating the malignant nodules from the benign ones using ultrasonography, whose interpretation results primarily depends on the subjectivity judgement of the radiologists. In this study, we propose a novel cascade deep learning model to achieve automatic objective diagnose during ultrasound examination for assisting radiologists in recognizing benign and malignant thyroid nodules. First, the simplified U-net is employed to segment the region of interesting (ROI) of the thyroid nodules in each frame of the ultrasound image automatically. Then, to alleviate the limitation that medical training data are relatively small in size, the improved Conditional Variational Auto-Encoder (CVAE) learning the probability distribution of ROI images is trained to generate new images for data augmentation. Finally, ResNet50 is trained with both original and generated ROI images. As consequence, the deep learning model formed by the trained U-net and trained Resnet-50 cascade can achieve malignant thyroid nodule recognition with the accuracy of $87.4 \%$, the sensitivity of $92 \%$, and the specificity of $86.8 \%$.
\end{abstract}

\section{Introduction}

Thyroid nodules often appear in a variety of thyroid diseases, including nodular goiter, thyroid adenoma and thyroid tumor. As one of the most common types of endocrine cancer, the incidence of thyroid cancer has increased globally in the past 30 years[1]. At present, the examination methods of thyroid nodules include palpation, serum thyroid stimulating hormone (TSH) level detection, ultrasound, and Fine Needle Aspiration (FNA) examination. Ultrasound is one of the most commonly used disease examination methods in clinical applications. It has the advantages of safety, convenience, non-invasiveness, and low price[2]. However, the ultrasound examination often has overlapping images of benign and malignant thyroid nodules, and sometimes misdiagnosis or missed diagnosis occurs. The diagnosis of benign and malignant relies on the expertise and clinical experience of the ultrasound doctor. The subjectivity of the doctor also has a great influence on the diagnosis. Computer-aided diagnosis (CAD) is a computerized technology that can be used as a second objective and accurate opinion to help doctors perform medical images by improving the accuracy and consistency of radiological diagnosis and reducing the reading time of radiological examinations Explanation and diagnosis. It is necessary to adopt the nodule segmentation and diagnosis system of thyroid ultrasound image based on computer-aided technology.
Deep learning model has been proved to be effective in medical image classification. Xiangchun Li et al. [3] integrated ResNet-50 and Dark-Net networks according to the performance of the two networks on the diagnosis of thyroid cancer. Lei Wang et al. [4]proposed to use the YOLOv2 network to detect and classify the benign and malignant thyroid nodules. The performance of the system in the diagnosis of thyroid nodules is comparable to the radiologists.

A large number of images are needed to train deep supervised convolutional network. However, large costs associated with the acquisition and labelling of clinical data have resulted in relatively small medical data sets for training these models. A good option is to use pretrained $\mathrm{CNNs}$ [5-7]. These models have learned general image features, such as edges and shapes, from a large amount of natural image data. By adding layers or retraining on the new data set, the parameters can be fine-tuned so that the network model has a better effect on the new data set. In order to increase the amount of data and make the network better trained, data augmentation methods are applied in pre-processing. Traditional methods such as random cropping, flipping, rotating, and distortion were often used to augment the thyroid nodules data[3,8-10], There is little work to determine which enhancement strategy can best capture medical image statistics and these methods cannot consistently improve the performance of deep models across different data sets.As a consequence, we propose a novel cascade deep learning model to achieve

\footnotetext{
* Corresponding author: wangr@buaa.edu.cn
} 
automatic objective diagnose during ultrasound examination for assisting radiologists in recognizing benign and malignant thyroid nodules.

The rest of this paper is organized as below. Section 2 summarizes the data and materials. Since then, the proposed method is introduced in detail in Section 3. Section 4 specifically outlines the experimental setup and results. Finally, Section 5 gives the conclusion.

\section{Data and materials}

All images of the dataset used in the study were collected using the ultrasound machine (Phillips, HITACHI, GE) with the probe frequency set as 5$17 \mathrm{MHz}$ and labeled by the doctors of Peking University Third Hospital. A total of 3771 thyroid nodule ultrasound images were collected from 2360 patients after surgery or Fine Needle Aspiration(FNA), among which 1316 are benign and 2455 were malignant. All the thyroid instances involved in this dataset have been examined via pathological examination and cover patients of different ages as well as nodules of different sizes.Fig.1(a)and(b)is an example of dataset.

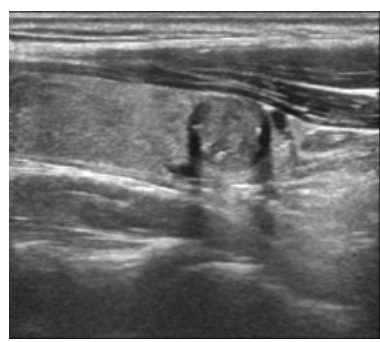

(a) The ultrasound image

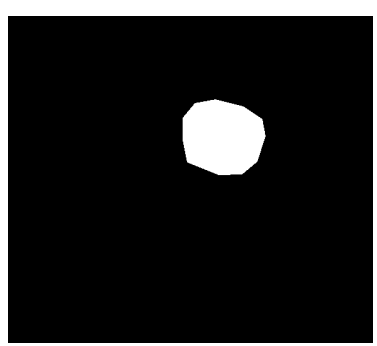

(b)the mask of thyroid nodule

Fig. 1. An example of dataset.

\section{Methods}

The method can be divided into three parts as shown in Fig.2. First, the thyroid nodule is automatically segmented by U-Net, and the ROI is extracted according to the segmentation results. Then the improves conditional variational auto-encoder network is used to perform data augmentation on the dataset. Finally, the pre-trained ResNet50 network is used to classify the benign and malignant nodules, and the classification effect is evaluated.

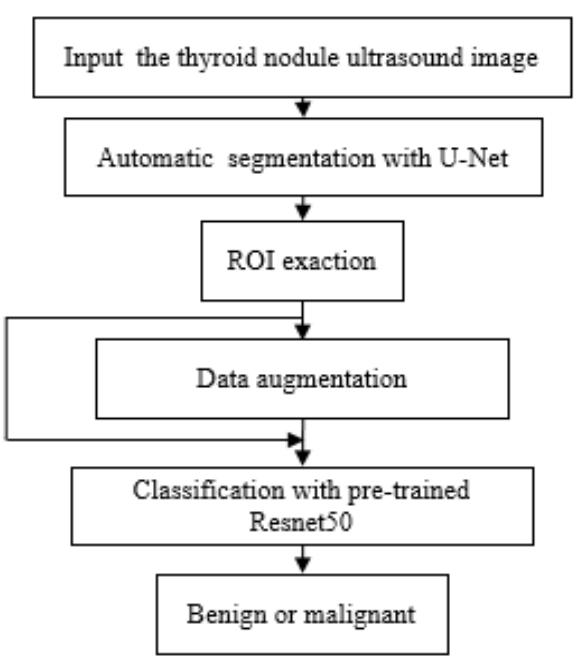

Fig. 2. The Proposed method

\subsection{Automatic segmentation with U-Net and ROI extraction}

U-Net[11] was first proposed for segmentation of neuronal structures in electron microscopic stacks and can be trained end-to-end from very few images. The architecture(Fig.3) consists of a contracting path to capture context and a symmetric expanding path that enables precise localization. Due to its lightweight and cascading deep features and shallow features, U-Net is effective in segmentation for semantically simple medical images.

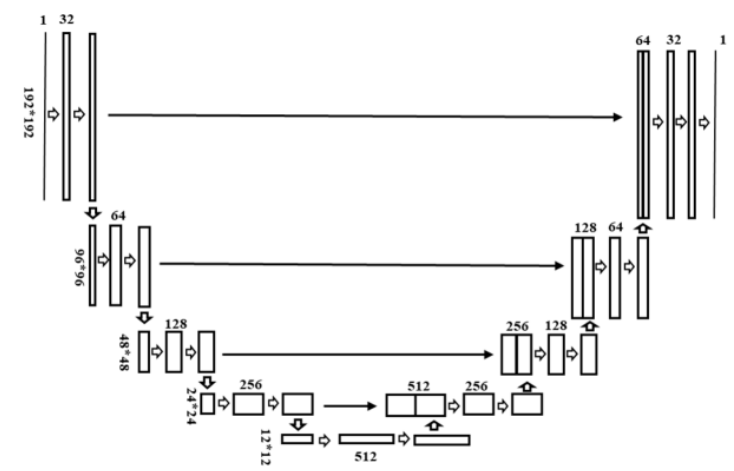

Fig. 3. The architecture of U-Net

A simplified U-Net is employed in our work. The contracting path consists of 4 convolutional blocks each of which performs two consecutive convolution operations with $16,32,64$ and 128 kernels of size $3 \times 3$ respectively and a $2 \times 2$ max pooling operation. In the symmetric expanding path, each convolutional block is followed by a $2 \times 2$ up sampling and a $2 \times 2$ convolution operation, Moreover, the feature maps are merged with those of the contracting path. In the last layer, the convolution kernel of $1 \times 1$ is used for convolution operation. Except that the output activation is sigmoid, the simplified U-Net activation functions are all rectified linear units (Relu). The loss function is defined as 


$$
\text { Loss }=1-\frac{2|\hat{y} ? y| \quad \varepsilon}{|\hat{y}|+|y|+\varepsilon}
$$

where $\hat{y}$ is output of network and $y$ is ground truth. $\varepsilon$ is a smoothing coefficient to avoid undefined loss, when both ground truth and output are zero.

An example of ROI is shown in Fig.4, corresponding to the original image in Fig.1(a)

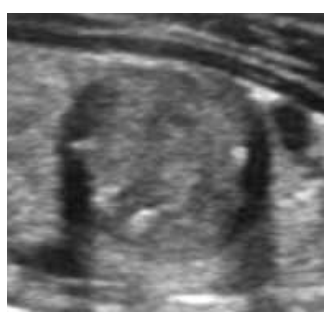

Fig. 4. An example of ROI

\subsection{Data augmentation}

\subsubsection{Variational Auto Encoder}

Variational Auto Encoder (VAE)[12] is a directed probability graph generation model composed of encoder $q_{\varphi}(z \mid x)$ and decoder $p_{\theta}(x \mid z)$. The encoder with parameter $\varphi$ maps the input $x$ to the latent variable $z$, and the decoder with parameter $\theta$ maps the latent variable to reconstructed data $\hat{x}$. The Parameter $\varphi$ and $\theta$ can be optimized through Auto-Encoding Variational Bayes (AEVB)[12] algorithm. The variational lower bound of VAE model is defined as:

$$
L(\theta, \varphi ; x)=-K L(q(z \mid x) \| p(z))+E_{q(z \mid x)}(\log p(x \mid z))
$$

where $K L(q(z \mid x) \| p(z))$ is the Kullback-Leibler divergence of $q(z \mid x)$ and $p(z)$. Equation (2) is also the objective function of VAE.The first term in Equation (2) uses KL divergence to minimize the difference between the encoder distributin $q_{\varphi}(z \mid x)$ and the prior distribution $p(z)$, The second item in Equation (2) represents the reconstruction loss. It makes the decoder learn to reconstruct the input data;Therefore, the goal of training VAE is to maximize the variational lower bound $L(\theta, \varphi ; x)$.

\subsubsection{Independent Conditional VAE[13]}

Conditional Variational AutoEncoder (CVAE)[14] is an extension of VAE , modeled by conditioning the encoder and decoder to label y. However, for data augmentation, it is preferred to have the encoding of images and the labels independent of each other. This allows one to control the characteristics of the image independently[13].This paper adopts the improve CVAE [14]where the lable $y$ has no affect on the latent variable $z$.The encoder maps the input $x$ into the latent variable $z$ and the decoder uses the labels $y$ and the latent variable to reconstruct the input $\hat{x}$.In order to balance the impact of $y$ and $z$ on $\hat{x}$, encoder parameterized by $\omega$ compresses y into a vector $\hat{y}$ with matching dimension with z.The variational lower bound of ICVAE is

$$
\begin{aligned}
L(\varphi, \theta, \omega, x, y)= & E_{q_{\varphi}(z \mid x)}\left[\log p_{\theta, \omega}(x \mid y, z)\right]-K L\left(q_{\varphi}(z \mid x) \| p(z)\right)+ \\
& \log p(y)-\log (y \mid x)
\end{aligned}
$$

If the batch size is large, $\log p(y)-\log (y \mid x)$ is negligible.

\subsection{The pre-trained ResNet-50[15]}

Transfer learning is widely used in medical images. Menegola et al.[16]conducted some experiments and compared fine-tuning training from scratch to pretraining network. The results showed that fine-tuning layer by layer based on specific applications can effectively understand the depth of tuning. Different data sizes also affect the number of layers of fine tuning. And it is concluded that no matter what the available data set size is, the fine-tuned CNN should be the first choice.

The model used to classify thyroid nodules in this paper is ResNet-50 pre-trained on ImageNet, which won the champion in ILSVRC2015. The proposal of ResNet50 successfully solves the problem of deep network degradation, enabling the network to achieve good performance and efficiency even in the case of deeper network layers. We fine-tune ResNet-50 on thyroid nodules dataset. To evaluate the reliability of the classifier, we further train Inception V3 and Mobile net.

\section{Experiments}

The experiments are accomplished on the Ubuntu 14.04 system with NVIDIA GeForce 1080 GPU. We use Keras/tensorflow to train the simplified U-Net for 80 epochs with batch size 16 . The optimization method used is Adam and learning rate is set $10^{-4}$. Besides, the number of training set, validation set and test set are 2771,500 and 500 respectively. We use dice $=\frac{2|\hat{y} ? y| \varepsilon}{|\hat{y}|+|y|+\varepsilon}$ to evaluate the segmentation performance. The simplified U-Net achieves dice 0.78 on the testing set.

In this paper,The distribution of $q_{\varphi}(z \mid x)$ is multivariate Gaussian distribution.The distribution of $p_{\theta, \omega}(x \mid y, z)$ is multivariate Bernoulli distribution. The ICVAE model used two fully connected layers symmetrically in the encoder and decoder, with 1024 and 512 neurons respectively. The activation function for each layer is $\tan \mathrm{H}$, except that the output is activated by the sigmoid function. The dimension of the latent variable is set to 10 .The ICVAE is trained for 100 epochs with the batch size 100 .

The classification results are obtained through gradual fine-tuning ResNet-50, that is, the parameters of the full connection layer are first trained, then the convolutional layer is released layer by layer, until all are released. The proportion of training set and test set is 
$8: 2$, and the proportion of benign and malignant samples of training set and test set is the same.

The classification result on the test set is shown in the Table 1. The accuracy is calculated by $\frac{T P+T N}{T P+F P+F N+T N}$, the sensitivity is calculated by $\frac{T P}{T P+F N}$, and the specificity is calculated by $\frac{T N}{F P+T N}$. As can be seen, ResNet50 outperforms InceptionV3 and MobileNet. The imbalance in the number of benign and malignant nodules in the original dataset resulted that the network could not accurately identify the benign samples as well as the malignant samples. After training the ICVAE, 1000 images were added to the to the training dataset to balance the number of benign and malignant nodules. The accuracy of ResNet50 on the augmented data set increases by 0.023 to 0.874 .

Table 1. Comparison of the classification metrics of the test data.

\begin{tabular}{|c|c|c|c|}
\hline Models & Accuracy & Sensitivity & Specificity \\
\hline $\begin{array}{c}\text { Inception- } \\
\text { V3 }\end{array}$ & 0.80 & 0.855 & 0.731 \\
\hline MobileNet & 0.838 & 0.902 & 0.756 \\
\hline ResNet 50 & 0.851 & 0.923 & 0.824 \\
\hline $\begin{array}{c}\text { Aug+ } \\
\text { ResNet 50 }\end{array}$ & 0.874 & 0.92 & 0.868 \\
\hline
\end{tabular}

\section{5 conclusion}

The classification of thyroid nodules is of great significance. This paper studies the Intelligent diagnosis of thyroid nodules. The overall process can be divided into three parts: ROI region acquisition, data augmentation, and nodule classification. Automatic segmentation of ROI areas with U-Net can not only help doctors to find the specific location of nodules but also provide margin, shape and aspect ratio of nodules which is important for diagnosis. We employed a generative network based on variational auto-encoders that can learn the distribution of the input for data augmentation and balancing the data set. Compared to no augmentation, the classification accuracy achieved through fine-tuning the pre-trained ResNet-50 increases by $2.3 \%$ on test set, which reveals the data augmentation strategy is effective.

\section{Acknowledgements}

This work was supported in part by a grant from National Natural Science Foundation of China (61673039).

\section{References}

1. P. Gabriella,F. Francesco,R. Concetto,S. Sebastiano,V. Riccardo, Worldwide Increasing
Incidence of Thyroid Cancer: Update on Epidemiology and Risk Factors, J. Cancer Epidemio, (2013)

2. K. Gul, D. Ozdemir, B. Korukluoglu, Preoperative and postoperative evaluation of thyroid disease in patients undergoing surgical treatment of primary hyperparathyroidism, Endocr Pract,16,7-13(2010).

3. X. Li, S. Zhang, Q. Zhang,et al.Diagnosis of thyroid cancer using deep convolutional neural network models applied to sonographic images: a retrospective,multicohort,diagnostic study ,The Lancet Oncology, 20(2),193-201(2019)

4. L.Wang, S. J. Yang, S. Yang,et al. Automatic thyroid nodule recognition and diagnosis in ultrasound imaging with the YOLOv2 neural network ,World Journal of Surgical Oncology, 17(1),12(2019)

5. Q. Guan, Y. Wang, J. Du, et al.Deep learning based classification of ultrasound images for thyroid nodules: a large scale of pilot study, Ann. Transl. Med, 7(2019).

6. J. Song,Y.Chai, H. Masuoka ,et al.Ultrasound image analysis using deep learning algorithm for the diagnosis of thyroid nodules ,Medicine, 98(15),e15133 (2019).

7. O. Moussa., H.Khachnaoui., R.Guetari.,et al.Thyroid Nodules Classification and Diagnosis in Ultrasound Images using Fine-Tuning Deep Convolutional Neural Network, INT. J. IMAG.SYST. TECH. (2019).

8. K. V. S. Sundar, K. T. Rajamani, S. S. S. Sai, Exploring Image Classification of Thyroid Ultrasound Images Using Deep Learning ,ISMACCVB, 1635-1641(2018)

9. M. Guo, Y. Du, Classification of Thyroid Ultrasound Standard Plane Images using ResNet-18 Networks ,ASID, 324-328(2019)

10. J. Wang, S. Li, W. Song, Learning from WeaklyLabeled Clinical Data for Automatic Thyroid Nodule Classification in Ultrasound Images, ICIP(2018.)

11. O. Ronneberger, P. Fischer, T. Brox, UNet:Convolutional Networks for Biomedical Image Segmentation,MICCAI,234-241(2015), ,

12. D. $\mathrm{P}$.Kingma , M.Welling. Auto-encoding variational bayes arXiv preprint arXiv:1312.6114(2013).

13. M. Pesteie, P. Abolmaesumi, and R. N. Rohling, Adaptive augmentation of medical data using independently conditional variational autoencoders,IEEE T. MED. IMAGING, 1-1(2019).

14. K. Sohn, X.Yan, H. Lee, et al. Learning Structured Output Representation using Deep Conditional Generative Models,ICNIPS( 2015)

15. K. He, X. Zhang, S. Ren, J. Sun, Deep Residual Learning for Image Recognition,CVPR,770778(2016). 
16. N. Tajbakhsh J.Y Shin ,S. R Gurudu et al., Convolutional Neural Networks for Medical Image Analysis: Full Training or Fine Tuning?,IEEE T. MED. IMAGING,35,1299-1312(2016). 